\title{
Improved evaluation of granular media flows using an X-ray scanning com- patible cone-plate setup
}

\author{
Zohreh Farmani ${ }^{1}$, Jing Wang ${ }^{2}$, Ralf Stannarius ${ }^{2}$, Martina Bieberle ${ }^{3}$, Frank Barthel ${ }^{3}$, Uwe Hampel ${ }^{3}$, and Joshua A. \\ Dijksman $^{1}$ \\ ${ }^{1}$ Physical Chemistry and Soft Matter, Wageningen University \& Research, Stippeneng 4, 6708 WE Wageningen, The Netherlands \\ ${ }^{2}$ Institute of Physics, Otto von Guericke University, Magdeburg, Germany \\ ${ }^{3}$ Inst. of Fluid Dynamics, Helmholtz-Zentrum Dresden-Rossendorf, Dresden, Germany
}

\begin{abstract}
To understand the typically heterogeneous flowing behavior of granular materials, it is important to combine flow tests with three-dimensional imaging. To probe the flow behavior of granular materials over a wide range of flow rates, it is imperative to be able to impose such flow rates in a well controlled manner while performing imaging tests that are compatible with all imposed flow rates. Achieving both flow control and bulk imaging capacity is challenging for a number of reasons. Here, we describe the design of a setup in which we are able to do imaging while imposing a constant overall shear rate on a granular material. We characterize the setup in which flow tests will be performed, which consists of a bottom-driven cone-plate or double-cone design. We show that the setup can be integrated in x-ray microtomography devices to aid particle tracking based flow measurements. The design is also compatible with typical rheometer setups. We also perform high speed imaging of a granular flow in an ultra-fast x-ray scanner, for which we provide proof-of-principle data in a simplified shear setup. The designed flow geometry is also compatible with said high speed imaging facility, where particle image velocimetry can be employed to extract quantitative flow field data.
\end{abstract}

\section{Introduction}

Granular flows are challenging to study experimentally for a number of reasons. One of the most important issues is that it is demanding to test constitutive equations by imposing "simple" boundary conditions like the constant strain rate conditions as imposed by a typical cone-andplate geometry: a granular material does not confine itself to the measurement gap like a fluid will do via its surface tension. To confine the shearing material to the measurement gap, one needs to close the system with a wall, complicating the flow field. Such geometries have been explored successfully however $[1,2]$ and can work effectively if one takes care of appropriate side wall flow conditions or confining pressure effects. Despite this progress, it is not clear what the induced flow profiles in such setups are. This limitation greatly impedes our understanding of granular flows and their continuum modeling efforts. In particular, the lack of flow structure data limits our ability to perform experiments in which we can test the role of individual microscopic parameters, such as particle deformation, shape or cohesion. To resolve these issues, it is important to design experimental setups in which we have access to the three-dimensional (3D) flow field while at the same time we retain control over, and insight in, the rheological (boundary) conditions of the flow. Here, we present one route towards such experimental progress, by describing a flow geometry that can impose system wide constant flow rates at the boundary, over a wide range of driving rates, while it is also compatible with slow and fast 3D imaging devices and can be equipped with, or used in conjunction with rheological instruments.

To highlight the challenge of probing the flow dynamics of granular materials, we consider the various options available in both slow and fast flow limits. Here we consider slow and fast as defined by the inertial or viscous inertial numbers [3]. To study slow flows one slowly moves a boundary of a container containing grains where the stresses and flow profiles become independent of the flow rate [4]. Shear bands then often localize near the moving boundaries. One may employ geometries such as the split-bottom Couette geometry or its variants [5] which can be used to impose very particular flow boundary conditions that induce wide shear zones [6] far away from any boundary. However, these flow profiles are fully three-dimensional and intricate, and stress conditions are difficult to impose. For more industrial contexts, Jenike, Schulze and many others have designed testers that exhibit narrow shear zones but well controlled stress boundary conditions [7]. Classical rheology setups such as plateplate [8] can impose boundary conditions over many orders of magnitude in flow rate, but here the shear zones are also often localized and thus spatially heterogeneous, all at least partially sensitive to wall effects. To consider faster flows limits, one can use inclined plane [9], avalanche flows [10] or hopper flows, or perform impact tests [11], all of which offer access to bulk flow behavior but only limited control of the flow profiles and bound- 
ary conditions. Finally, rotating drum experiments offer good control over the overall flow rate, but often display intricate flow behavior, ranging from stick-slip to highly heterogeneous flow, for example for cohesive particle systems. In particular, heterogeneous flow profiles are not desirable as they limit the applicability of the geometry for the study of faster flows. Typical high speed imaging applications use direct cross-sectional imaging methods such as index matched laser sheet imaging [12] or MRI [13], as the imaging rate must be comparable to the displacement rate of particles. Mapping fully three dimensional flows is then too time consuming, and this puts limits on the complexity of the flow structure one can accurately handle in experiments.

To study granular flows with reasonable control over the boundary conditions and with simple quasi- two dimensional flow profiles, one is therefore limited to a coneplate (CP) or double-cone (DC) geometry: shear and normal stresses on bottom and top walls can in principle be controlled, while the imposed strain rate is independent of the radial position. The flow profiles induced have a radial symmetry at least in steady state, and perhaps even an up-down symmetry in double-cone geometry when gravity can be neglected. Such flow profiles can be measured accurately and relatively easily over a wide range of flow rates. For this reason, much progress has already emerged from the use of such setups $[1,2]$. However, such flow geometries have not yet been combined with extensive flow imaging over a wide range of flow rates. In this regard, it is of paramount importance to design a new flow setup which helps us to have a full understanding of a flow field by visualizing the internal dynamics of the flow. Thus, we designed a shear setup that can be used in many 3D imaging facilities and is compatible with slow and fast flows. Additionally, the flow geometry must be compatible with (custom) rheological measurement systems. As there are already many slow 3D imaging methods available, the main challenge is to have a setup that allows for fast flow imaging. Laser sheet imaging and MRI are limited to fully transparent or non-magnetic, fluid-containing materials, greatly limiting material choice and setup design space. Here we make use of the recently developed capacity for high speed slice imaging based on x-ray technology. Ultrafast X-ray computed tomography is nowadays possible at the Helmholtz-Zentrum Dresden-Rossendorf (ROFEX) [14]. Contrary to conventional tomography systems, the ROFEX systems do not get radiographic projections by mechanical rotation of the object or source-detectorcompound. Instead, an electron beam is focused towards a circular target and at the same time periodically deflected with high frequency. In this way, a focal spot moving along the target, i.e. an X-ray source rotating around the object, is generated in timescales on the order of submilliseconds. Therefore, the device is able to take real-time 2D cross sectional images during dynamic experiments. Previous studies prove the potential of this scanner for dynamic tests $[15,16]$. First we will explain the new design of the flow geometry, then present slow but detailed 3D imaging study results of arrested states, and after that

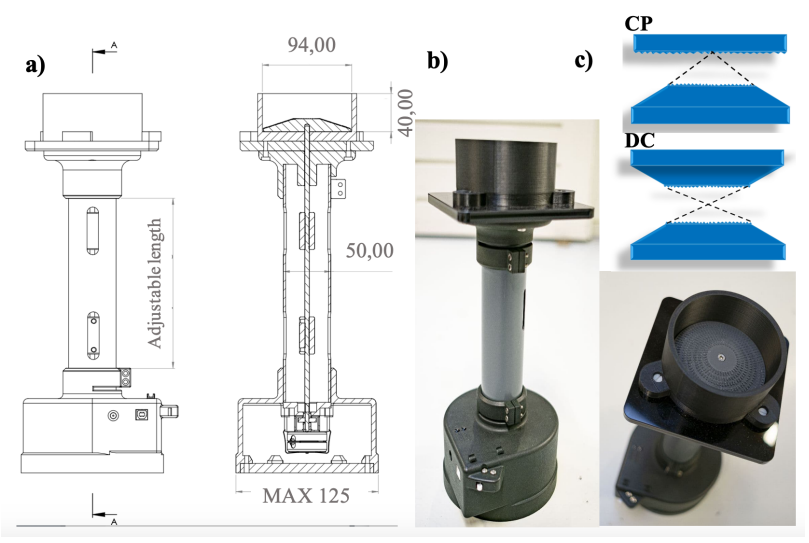

Figure 1. (a) Schematic of the new shearing setup. The 2D sketches indicate the dimensions (in $\mathrm{mm}$ ) in detail, (b) Actual images of the setup, part of which was constructed with 3D printing techniques, and (c) CP (Cone/Plate) and DC (Double Cone) configurations.

we present first fast in-plane shear flow data from ROFEX measurements.

\section{Experimental Setup}

The main design idea of the CP/DC flow geometry with imaging and rheological capabilities is shown in Fig. 1. In this figure, we show the whole view of the setup that we have designed in order to do shearing and imaging at the same time. Particles of diameter $1.5 \mathrm{~mm}$ to about $4 \mathrm{~mm}$ can be used to fill the space between two rough cones. The cylindrical cell has an outer diameter of $104 \mathrm{~mm}$, inner diameter of $94 \mathrm{~mm}$, and height of $50 \mathrm{~mm}$, cones with a diameter of $92 \mathrm{~mm}$ and an angle of around 13, and bottom plate are $3 \mathrm{D}$ printed. The bottom cone is fixed to the bottom of the cell by means of an o-ring and is connected to the motion rod which is rigidly attached to the stepper motor to the bottom cone using two connectors. While the bottom cone rotates with a certain angular speed as set by the stepper motor, the upper cone is set to be at a fixed height. Alternatively, the top cone can be replaced with a disk for an (inverted) CP geometry. In order to be sure that all the particles are well contained between the cones, and have not been trapped between the tips of the cones, we make the cone tips flat at a distance of $1 \mathrm{~mm}$ below the apex. This gap size can be adjusted based on the particle size used. In addition, the gap between the cone and sidewalls should be equal or less than $1 \mathrm{~mm}$ from each side. We use a geared McLennan P542-M48 Series stepper motor with a maximum torque of $1 \mathrm{Nm}$. The torque range is chosen with the consideration that for granular materials, one needs to control or measure the ratio of the normal and shear stress, as this ratio ranging from 0 to 1 captures the effective friction coefficient of the flowing material. With a cone or plate radius of the order of $0.1 \mathrm{~m}$ and a maximum torque of $1 \mathrm{Nm}$, a friction coefficient of order unity is reached at a normal stress of $\approx 1 \mathrm{kPa}$, far below the elastic constant of particles of interest. Only 
in hydrogel spheres a significant compression could be induced if desired [12]. The motor can be operated with digital control over position and speed. This particular stepper motor needs 1200 steps per revolution, allowing for smooth rotation, although microstepping may be needed for improved operation. Rheological measurements can be combined with the flow cell in two ways: we design the setup in such a way that the top cone/plate can be held by a Futek MBA500 sensor to give access to both normal and shear stresses acting on the top plate. Additionally, the flow cell can be mounted on a standard Anton Paar MCR body with a custom made flange. We can use particles immersed in water as the setup is waterproof: the rotating shaft can be sealed with a lip seal. These two stress measurement devices allow for the use of a wide range of materials, from soft hydrogel beads to hard grains, as the torque sensitivities of the respective devices give us access to shear and normal stress of up to $1 \mathrm{kPa}$. Note that the side walls of the container can in principle be made soft or elastic to allow for pressure control. The setup is designed in such a way it can be used in various X-ray tomography devices. The cell is designed to be compatible with the following (1) a commercially available $\mathrm{x}$-ray microtomographer with a $40 \mathrm{kV} / 320 \mathrm{~W}$ microfocus CT system (Phoenix V|Tome $\mid \mathrm{X}$ ) available in Wageningen University; (2) the custom built high resolution micro-CT in University of Grenoble Alpes [17]; (3) the ultra-fast X-ray scanner in Rossendorf (ROFEX), Germany.

\section{Results and Discussions}

\subsection{Slow flow imaging capacity}

We used a cone-plate version of our setup to 3D image a static assembly of spherical Teflon particles with a diameter of $4.7 \mathrm{~mm}$. Importantly, we used that geometry to check whether the cone will introduce imaging artifacts in the 3D image reconstruction algorithms: the cone introduces a large X-ray absorbing mass in the center of the structure that in principle can introduce artefacts such as motion artifacts, twin/double exposure, crescent-shaped black and white lines, clear spots, and interfering with particles, as the cone is made out of nylon. We could confirm from the tomograms obtained that the cone does not present significant image distortion issues. We used the $\mathrm{X}$-ray microtomographer with a spatial resolution down to $55 \mu \mathrm{m}$, an imaging time scale of 30 minutes, with voltage of $40 \mathrm{kV}$ and current of $150 \mu \mathrm{A}$. After reconstruction and processing the images, we obtained the segmented cross section of the grains seen in Fig. 2. Image analysis was performed in Avizo. Note that the particles used here were large for the dimensions of the system, and the gap size between the top plate and cone was held large enough to ensure a complete filling of the space with particles. We used both teflon and nylon grains in the 3D imaging, as can be observed from the different gray values of some particles in Figs. 2d-f.
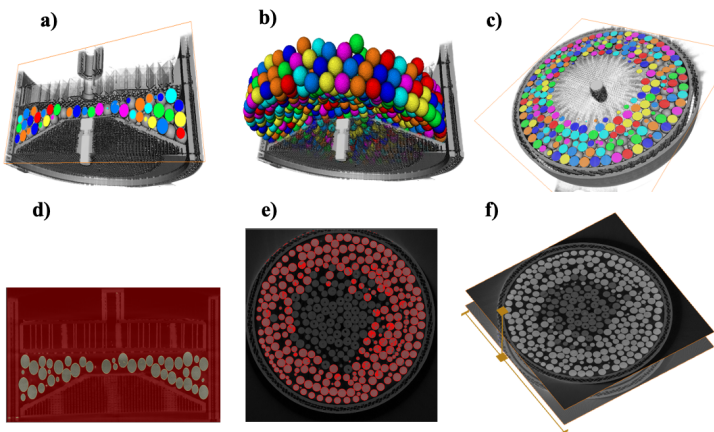

e)

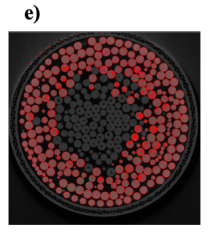

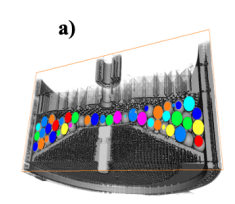

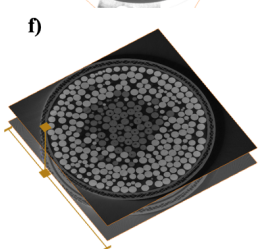

Figure 2. Segmented 3D images of spherical teflon particles (4.7 $\mathrm{mm}$ ) in the shear cell described in Fig. 1. Colors represent individual labeled grains. (a) cross section of the particles inside a cone-plate shear setup. (b) a full 3D view of the particles. (c) Segmented spherical particles, top view. (d) 2D section from scanning of our second material after rotation. (e), (f) Teflon and nylon grains show up with a different gray level, as they have a different $\mathrm{x}$-ray absorption coefficient.

\subsection{Fast flow imaging capacity}

To demonstrate the fast imaging capacity of the ROFEX facility for granular flows driven in shear cells, we imaged in preliminary test experiments the flow generated by a plate-plate system. The ROFEX facility allows for the simultaneous imaging of two slices with sub-millisecond time resolution. We employed this technique to record the shear of hard grains in a cylindrical container of $94 \mathrm{~mm}$ inner diameter. A rotating top plate is placed $22 \mathrm{~mm}$ above a resting bottom plate. The two imaged slices can be chosen at any height in the sheared zone, with a mutual distance of $11 \mathrm{~mm}$. We determined some aspects of the internal flow fields by image analysis. Tests were performed on a 4:1 glass/aluminum grain mixture with an average particle diameter of $2 \mathrm{~mm}$. With the resolution of $1 \mathrm{~mm} / \mathrm{pixel}$ chosen here, the individual particles could not be identified, but image contrast should allow for image velocimetry. Our experiments revealed a feature of the ROFEX system that needs to be taken into account when imaging the flow of granular materials in the imaging plane. While the electron beam scanning is fast, particle displacements during acquisition may lead to reconstruction artifacts. This limits the rotation rate for useful tomographic results to roughly $10 \mathrm{rpm}$ for the system studied here. The use of labeled tracer grains would improve the situation substantially. With tracer particles, particle tracking instead of PIV would be possible. The problem is that particles with larger X-ray contrast also have larger mass densities, these two quantities are more or less related. Thus, using heavier markers would induce segregation during shear, which is undesirable. Nevertheless, one can employ image analysis techniques to obtain some flow information from the comparison of reconstructed consecutive 2D images obtained during steady driving. We extracted an intensity profile along a tangential cut of the tomograms using Matlab; the results of which are combined in a kymograph in Fig. 3d. The figure displays an line intensity profile from the reconstructed images $1 \mathrm{~mm}$ below the rotating 

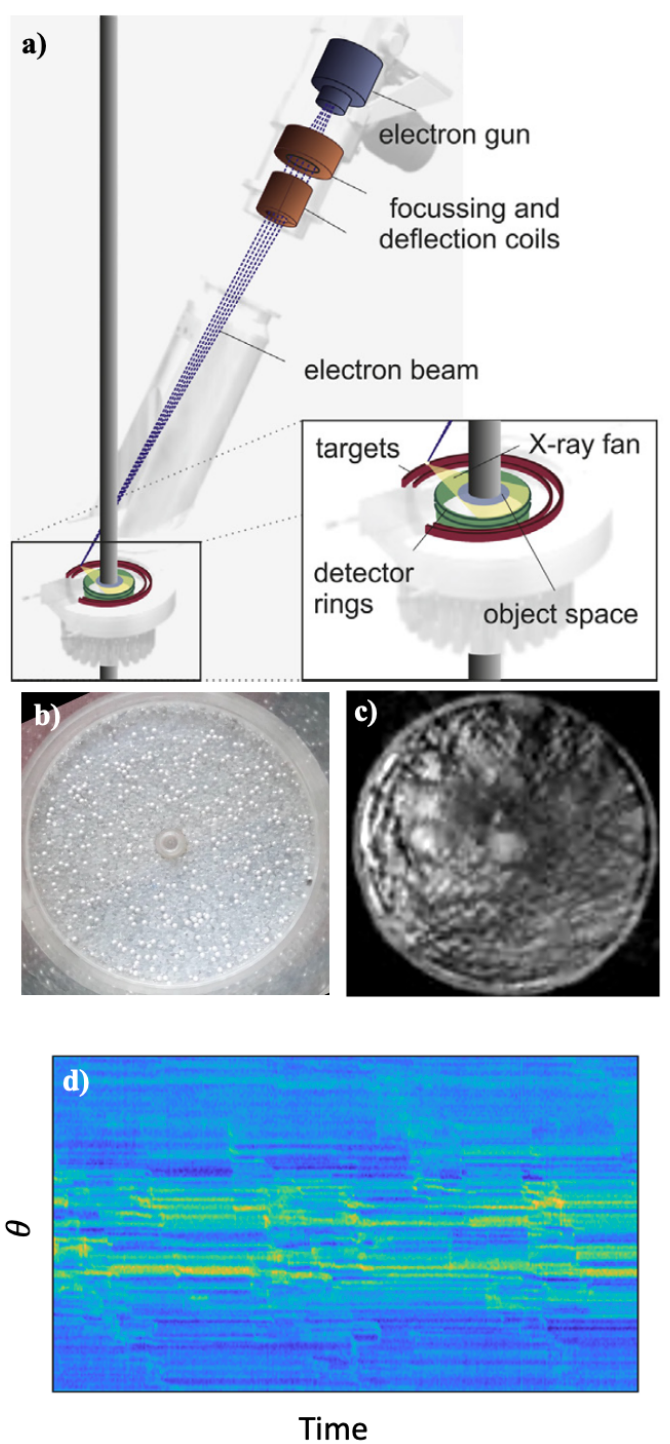

Figure 3. (a) Schematic design of the ROFEX imaging facility. Image taken from Ref. [14], (b) Optical top image of the plateplate shear cell and (c) tomographic cut $1 \mathrm{~mm}$ below the upper plate, obtained during the shear experiment with the bottom plate at rest and the top plate rotating with $9 \mathrm{rpm}\left(\omega=0.94 \mathrm{~s}^{-1}\right)$. Inner diameter of the plate $94 \mathrm{~mm}$, gap between plates $22 \mathrm{~mm}$, (d) Kymograph of the 2D section.

top plate, extracted as a function of the $\theta$ coordinate at a radius of $32 \mathrm{~mm}$ for the entire duration of 20,000 images (20 s). The $\theta$ and time dependence of the intensity profile dynamics strongly suggests that flow in the bulk of this plate-driven medium is heterogeneous, with local stickslip events taking place. In this experiment, the plate-plate geometry generated a localized shear zone very close to the moving boundary. The simultaneous imaging of two slices was therefore not effective: while the top slice exhibited features of the displacement fields, the second slice $11 \mathrm{~mm}$ below the first one showed no motion of the granular material. The complete shear zone was located above that slice. This was confirmed qualitatively by an optical inspection through the transparent side walls.

\section{Conclusions}

In summary, we have presented a setup which is able to realize slow and fast shearing and imaging at the same time and can give us comprehensive information on the flow field of the sheared grains. The preliminary real-time data obtained from high-speed imaging provide a promising proof-of-concept for this approach. We find it advantageous to change the geometry to the typical rheological geometries, such as cone-plate and double-cone, because the shear distribution is affected by the choice of geometry.

\section{Acknowledgments}

This project received funding from the European Union's Horizon 2020 research and innovation program under the Marie Skłodowska Curie grant agreement No 812638. Diego Sancho Martinez is acknowledged for designing the plate-geometry setup and participating in experiments.

\section{References}

[1] F. Boyer, É. Guazzelli, O. Pouliquen, Phys. Rev. Lett. 107, 188301 (2011)

[2] J.C. Tsai, M.R. Chou, P.C. Huang, H.T. Fei, J.R. Huang, Soft Matter 16, 7535 (2020)

[3] J.A. Dijksman, E. Wandersman, S. Slotterback, C.R. Berardi, W.D. Updegraff, M. van Hecke, W. Losert, Phys. Rev. E 82, 060301 (2010)

[4] R. Mani, D. Kadau, D. Or, H.J. Herrmann, Phys. Rev. Lett. 109, 248001 (2012)

[5] J.A. Dijksman, M. van Hecke, Soft Matter 6, 2901 (2010)

[6] S. Luding, Granul. Matter 10, 235 (2008)

[7] D. Schulze, Powders and bulk solids:Behaviour, Characterization, Storage and Flow, Springer (2008)

[8] K. Lu, E. Brodsky, H. Kavehpour, J. Fluid Mech. 587, 347 (2007)

[9] G. MiDi, Eur Phys J E 14, 341 (2004)

[10] P. Tegzes, T. Vicsek, P. Schiffer, Phys. Rev. Lett. 89, 094301 (2002)

[11] T. Faug, Can. Geotech. J. 58, 114 (2020)

[12] J.A. Dijksman, N. Brodu, R.P. Behringer, Rev. Sci. Instr. 88, 051807 (2017)

[13] R. Stannarius, Rev. Sci. Instr. 88, 051806 (2017)

[14] M. Bieberle, F. Barthel, Chem. Eng. J. 285, 218 (2016)

[15] S. Waktola, A. Bieberle, F. Barthel, M. Bieberle, U. Hampel, K. Grudzień, L. Babout, Exp. Fluids 59, 69 (2018)

[16] R. Stannarius, D. Sancho Martinez, T. Börzsönyi, M. Bieberle, F. Barthel, U. Hampel, New J. Phys. 21, 113054 (2019)

[17] E. Andò, S.A. Hall, G. Viggiani, J. Desrues, P. Bésuelle, Acta Geotech. 7, 1 (2012) 